\section{Bewegung bei Krebs im fortgeschrittenen Stadium: Was sind die Motive der Betroffenen und welche Hindernisse gibt es?}

\section{Originalpublikation}

Frikkel J, Götte M, Beckmann M, et al. Fatigue, barriers to physical activity and predictors for motivation to exercise in advanced Cancer patients. BMC palliative care 2020; 19: 1-11 [1].

\section{Hintergrund}

Wenngleich die Pioniere der onkologischen Sport- und Bewegungstherapie von der Deutschen Sporthochschule Köln ihrerzeit noch auf massiven Gegenwind stießen, Krebspatienten mit Bewegung zu versorgen [2], liegt mit über 700 klinischen Studien inzwischen eine gewaltige Evidenz zugunsten Bewegung in der Onkologie vor [3]. Für die Patientenversorgung hat sich insoweit vor allem ein nebenwirkungsorientierter Ansatz [4] bewährt, wie er bspw. auch im Konzept der onkologischen Trainings- und Bewegungstherapie verfolgt wird [5], in welchem Bewegungsfachkräfte für die Betreuung onkologischer Patienten qualifiziert werden. Eine in diesen Bewegungskonzepten häufig adressierte und mit dem Tumor und seiner Therapie assoziierte Nebenwirkung ist die cancer related fatigue (CRF) [5]. Die CRF zeichnet sich durch eine dauerhafte $\mathrm{Er}$ schöpfung aus, welcher die Patienten sich trotz Erholungsphasen nicht entledigen können und die durch eine schwere Antriebslosigkeit weiter verstärkt wird. Bislang existieren keine wirksamen pharmakologischen Behandlungskonzepte zur Bekämpfung der CRF, und auch das National Comprehensive Cancer Network verweist in seinen Guidelines auf nicht-pharmakologische Interventionsstrategien [6]. Körperliche Aktivität und körperliches Training als Beispiele nichtpharmakologischer Interventionen konnten ihre Effektivität in einer Vielzahl randomisiert kontrollierter Studien unter Beweis stellen und ihre Überlegenheit gegenüber pharmakologischen Interventionen in einem Umbrella Review, einer systematischen Übersichtsarbeit, welche die Effektivität verschiedener Interventionen miteinander vergleicht, absichern [7]. Die positiven Effekte, welche körperliche Aktivität und körperliches Training für Krebspatienten bereithalten, sind jedoch nicht nur auf die CRF begrenzt, sondern umfassen etliche weitere Nebenwirkungen, die mit der Krebserkrankung und ihrer Therapie assoziiert sind [3, 9]. Hierbei ist jedoch wichtig anzumerken, dass der Großteil dieser Evidenz von Patienten mit nicht-metastasierten Tumorstadien stammt und vergleichsweise wenige Daten von Patienten mit fortgeschrittenen Krebserkrankungen vorliegen. Dennoch haben Dittus und Kollegen [10] in einer systematischen Übersichtsarbeit die Wirksamkeit körperlicher Aktivität auch bei fortgeschrittenem Tumorstadien nachgewiesen. In den eingeschlossenen Studien wurde in 14 von 19 Fällen die kardiovaskuläre Leistungsfähigkeit, in 11 von 14 Studien die Kraftfähigkeit und in 9 von 9 Arbeiten die körperliche Funktionsfähigkeit verbessert. Ferner wurde in 11 der 19 identifizierten Studien eine Verbesserung der Fatigue-Symptomatik beobachtet, was die Autoren zur Schlussfolgerung führt, dass körperliches Training für Menschen mit fortgeschrittener Krebserkrankung als Maßnahme verstanden werden sollte, durch welche weitere Gesundheitskomplikationen verhindert werden können. Hierbei ist anzumerken, dass in den z. B. 8 verbleibenden Studien, in welchen die Fatigue untersucht wurde, keineswegs eine substanzielle Vergrößerung der Fatigue beobachtet wurde, sondern der Mittelwertsunterschied zwischen der Interventions- und Kontrollgruppe lediglich die statistische Signifikanz verfehlte.

Trotz der erdrückenden, auf mehr als 700 klinischen Studien basierenden [6] Evidenzlage zugunsten körperlicher Aktivität und körperlichen Trainings über alle Krebsstadien hinweg, sind Krebspatienten in der Regel inaktiv. Beispielsweise untersuchten Lin und Kollegen in ihrer systematischen Übersichtsarbeit, in welcher das Aktivitätsniveau von insgesamt 1600 gynäkologischen Krebspatientinnen erfragt wurde, inwieweit die WHO-Empfehlung von 150 Minuten pro Woche moderater bzw. 75 Minuten wöchentlicher intensiver körperlicher Aktivität erreicht wird. Die Analysen zeigten, dass 2 Jahre nach Diagnosestellung lediglich 9\% der Studienteilnehmerinnen den WHO-Empfehlungen gerecht wurden [11]. Wenig überraschend zeigen Menschen mit fortgeschrittenen Tumorerkrankungen ebenfalls sehr niedrige Aktivitätswerte. In einem gemischten Patientenkollektiv konnte bei den vergleichsweise fittesten Patienten (ECOG-Score 0 und 1) per Akzelerometrie eine mediane Schrittzahl von etwas unter 4000 Schritten pro Tag beobachtet werden.

Bei dieser Diskrepanz aus wissenschaftlicher Evidenz und Handlungsrealität stellt sich unmittelbar die Frage, mit welchen Barrieren gegenüber körperlicher Aktivität sich Krebspatienten konfrontiert sehen. Auch zu dieser Frage liegen bereits einige Studien vor, die allerdings nur Patienten mit nicht-metastasierten Tumorstadien untersuchten und daher nur bedingt auf metastasierte Patienten übertragbar sind.

\section{Motive und Barrieren bzgl. körperlicher Aktivität bei metastasierten Krebspatienten}

Um verlässliche Aussagen über die Motive und Barrieren dieses Patientenkollektivs gegenüber körperlicher Aktivität treffen zu können, die dazu befähigen sollen, Strategien zu entwickeln, mithilfe derer Patienten mit fortgeschrittenem Tumorstadium zu mehr körperlicher Aktivität motiviert werden können, haben Frikkel und Kollegen [1] 141 Patienten mit fortgeschrittener Tumorerkrankung im Alter von 26 bis 83 Jahren (MW = 60 Jahre) befragt. Von diesen $141 \mathrm{~Pa}$ tienten gaben 110 an, körperlich inaktiv zu sein, die verbleibenden 31 Patienten waren mindestens 1-mal pro Woche aktiv.

Als häufigste Barriere (77\%) wurde von den Befragten genannt, dass sie sich durch die Tumortherapie geschwächt fühlen, 72 \% bzw. $70 \%$ der Patienten berichten zudem von Schlafstörungen und CRF. Interesse und Desinteresse an körperlicher Aktivität bekunden jeweils die Hälfte der Untersuchungsteilnehmer, $41 \%$ berichten, dass sie bereits vor der Diagnose körperlich inaktiv waren. Etwa 25\% der Kohorte haben Zweifel an der Wirksamkeit körperlicher Aktivität auf die Lebensqualität. Angst vor möglichen Adverse Events bei der Durchführung körperlichen Trainings wird von einem pro vier Befragten geäußert.

Der Gruppenvergleich der körperlich inaktiven und der aktiveren Patienten zeigt außerdem eine enge Assoziation zur Anzahl der Komorbiditäten. Ferner deuten die Ergebnisse auf die bereits vielfach beobachtete höhere Motivation von Frauen hinsichtlich eines körperlich aktiven Lebensstils hin. So lag der An- 
teil der Brustkrebspatientinnen bei den Aktiven höher als in der inaktiven Gruppe.

Zusammenfassend lässt sich festhalten, dass sich Patienten mit fortgeschrittenem Krebsstadium mit einer Vielzahl an Barrieren gegenüber körperlicher Aktivität konfrontiert sehen. An erster Stelle sind hierbei der Verlust körperlicher Leistungsfähigkeit und Erschöpfungssyndrome zu sehen. Paradoxerweise ist gerade körperliche Aktivität das beste Mittel, um diesen Beschwerden zu begegnen. Durch diese Wechselwirkung von körperlicher Aktivität und CRF bzw. reduzierter körperlicher Leistungsfähigkeit ergibt sich eine große Herausforderung, um nicht nur effektive, sondern auch realisierbare Bewegungsmaßnahmen zu etablieren. Hierzu muss zunächst der Teufelskreis aus körperlicher Inaktivität, körperlicher Dekonditionierung und CRF durchbrochen werden und eine Heranführung an körperliche Aktivität geschehen. Zudem existieren inzwischen evidenzbasierte Leitlinienempfehlungen, wie die Fatigue-Symptomatik und die reduzierte körperliche Leistungsfähigkeit bewegungstherapeutisch anzugehen sind [9]. Die Gretchenfrage, wie Patienten initial in Bewegungsprogramme gebracht, dann aber auch dabei gehalten können, bleibt weiterhin offen. In einer Übersichtsarbeit von Ormel und Kollegen [12], die sich genau mit dieser Frage auseinandersetzten, wurden hierfür mehrere Faktoren identifiziert, welche einen positiven Einfluss auf die Adhärenz der Patienten besitzen. Als relativ naheliegend wurde die grundsätzliche Motivation der Patienten als wichtiger Einflussfaktor genannt. Basierend auf etablierten gesundheitspsychologischen Modellen kann es daher sinnvoll sein, seitens der Patienten wichtige Determinanten der Motivation bzw. Intention, z. B. die Einstellung zur Bewegung oder eine positive subjektive Norm, zu stärken. Anders ausgedrückt: Das Gesundheitsfachpersonal muss dazu angehalten werden, explizit darauf hinzuweisen, wie wichtig körperliche Aktivität und körperliches Training für die Patienten ist. Weiterer bedeutsamer Erfolgsfaktor für die Adhärenz von Krebspatienten an Bewegungsprogrammen ist die Wohnortnähe des Bewegungsanbieters. Dem Patienten soll es also logistisch einfach gemacht werden, ein körperliches Training aufzunehmen. Durch die substanziell reduzierte Leistungsfähigkeit, Nebenwirkungen der Tumorthera- pie und Komorbiditäten von Patienten mit Krebs im Allgemeinen und Patienten mit fortgeschrittener Erkrankung im Besonderen sollte hier ein qualitätsgesicherter Prozess im Hintergrund stehen, der eine gute Betreuung der Patienten gewährleistet. Voraussetzung hierfür ist zuvorderst eine flächendeckende und hochwertige Anbieterqualifikation. Durch den Deutschen Verband für Gesundheitssport und Sporttherapie (DVGS e.V.) und seine etwa 3000 Mitglieder könnte dies theoretisch gewährleistet werden. Ein weiterer Baustein ist die Verknüpfung von Kliniken, Rehabilitationseinrichtungen und Bewegungsförderern bzw. -anbietern, um eine kontinuierliche, bewegungstherapeutische Versorgung der Patienten und eine lückenlose Schnittstellenüberbrückung zu ermöglichen. Das Netzwerk OnkoAktiv [13] hat sich genau dieser Aufgabe gestellt und bereits in 8 Bundesländern damit begonnen, das Vorhaben in die Tat umzusetzen. Für weitere Informationen zum Netzwerk OnkoAktiv, der onkologischen Bewegungstherapie im Allgemeinen und kostenfreien Informationsmaterialien sei an dieser Stelle auf die Homepage unterwww.netzwerk-onkoaktiv.de verwiesen.

\section{ZUSATZINFO}

Dieser Beitrag wurde bereits im Februar 2021 veröffentlicht unter https://dvgs.de/de/features/blog.html

Literatur

[1] Frikkel J, Götte M, Beckmann M et al. Fatigue, barriers to physical activity and predictors for motivation to exercise in advanced Cancer patients. BMC palliative care 2020; 19: 1-11

[2] Köppel M. Effekte körperlichen Trainings auf Lymphödeme bei Brustkrebs: Eine systematische Übersichtsarbeit. B\&G Bewegungstherapie und Gesundheitssport 2018; 34: 134-135

[3] Christensen JF, Simonsen C, Hojman P. Exercise training in cancer control and treatment. Comprehensive Physiology 2011; 9: 165-205

[4] Wiskemann J, Scharhag-Rosenberger F. Nebenwirkungsorientierte Behandlungspfade für die bewegungstherapeutische Betreuung onkologischer Patienten. B\&G Bewegungstherapie und Gesundheitssport 2014; 30: 146-150
[5] Baumann FT, Hallek M, Meyer ] et al. Onkologische Trainings-und Bewegungstherapie (OTT). DMW-Deutsche Medizinische Wochenschrift 2015; 140: 1457-1461

[6] Weis ]. Cancer-related fatigue: prevalence, assessment and treatment strategies. Expert review of pharmacoeconomics \& outcomes research 2011; 11: 441-446

[7] Berger AM, Mooney K, Alvarez-Perez A et al. Cancer-related fatigue, version 2.2015. Journal of the National Comprehensive Cancer Network 2015; 13: 1012-1039

[8] Mustian KM, Alfano CM, Heckler C et al. Comparison of pharmaceutical, psychological, and exercise treatments for cancerrelated fatigue: a meta-analysis. JAMA oncology 2017; 3: 961-968

[9] Campbell KL, Winters-Stone KM, Wiskemann ] et al. Exercise guidelines for cancer survivors: consensus statement from international multidisciplinary roundtable. Medicine \& Science in Sports \& Exercise 2019; 51: 2375-2390

[10] Dittus KL, Gramling RE, Ades PA. Exercise interventions for individuals with advanced cancer: a systematic review. Preventive medicine 2017; 104: 124-132

[11] Lin K-Y, Edbrooke L, Granger CL, Denehy L, Frawley HC. The impact of gynaecological cancer treatment on physical activity levels: a systematic review of observational studies. Brazilian journal of physical therapy 2019; 23: 79-92

[12] Ormel H, Van Der Schoot G, Sluiter W et al. Predictors of adherence to exercise interventions during and after cancer treatment: a systematic review. Psychooncology 2018; 27: 713-724

[13] Wiskemann J, Köppel M, Biazeck B. Sport und Bewegung nach Krebstherapie-OnkoAktiv als ein Beispiel. Der Gastroenterologe 2019; 14: 368-374

\section{Korrespondenzadresse}

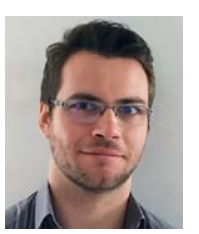

\section{Maximilian Köppel}

Deutscher Verband für

Gesundheitssport und

Sporttherapie e.V.

Vogelsanger Weg 48

50354 Hürth-Efferen

Deutschland

Maximilian.koeppel@outlook.de 